\title{
Review Article \\ Gab Docking Proteins in Cardiovascular Disease, Cancer, and Inflammation
}

\author{
Yoshikazu Nakaoka and Issei Komuro \\ Department of Cardiovascular Medicine, Graduate School of Medicine Osaka University, 2-2 Yamadaoka, \\ Suita, Osaka 565-0871, Japan \\ Correspondence should be addressed to Yoshikazu Nakaoka; ynakaoka@cardiology.med.osaka-u.ac.jp
}

Received 14 May 2012; Accepted 11 December 2012

Academic Editor: Masanori Aikawa

Copyright (C) 2013 Y. Nakaoka and I. Komuro. This is an open access article distributed under the Creative Commons Attribution License, which permits unrestricted use, distribution, and reproduction in any medium, provided the original work is properly cited.

\begin{abstract}
The docking proteins of the Grb2-associated binder (Gab) family have emerged as crucial signaling compartments in metazoans. In mammals, the Gab proteins, consisting of Gab1, Gab2, and Gab3, are involved in the amplification and integration of signal transduction evoked by a variety of extracellular stimuli, including growth factors, cytokines, antigens, and other molecules. Gab proteins lack the enzymatic activity themselves; however, when phosphorylated on tyrosine residues, they provide binding sites for multiple Src homology-2 (SH2) domain-containing proteins, such as SH2-containing protein tyrosine phosphatase 2 (SHP2), phosphatidylinositol 3-kinase regulatory subunit p85, phospholipase $\mathrm{C} \gamma$, Crk, and GC-GAP. Through these interactions, the Gab proteins transduce signals from activated receptors into pathways with distinct biological functions, thereby contributing to signal diversification. They are known to play crucial roles in numerous physiological processes through their associations with SHP2 and p85. In addition, abnormal Gab protein signaling has been linked to human diseases including cancer, cardiovascular disease, and inflammatory disorders. In this paper, we provide an overview of the structure, effector functions, and regulation of the Gab docking proteins, with a special focus on their associations with cardiovascular disease, cancer, and inflammation.
\end{abstract}

\section{Introduction}

The mammalian Grb2-associated binder (Gab) proteins are homologs of Drosophila DOS (Daughter Of Sevenless) and Caenorhabditis elegans SOC-1 (Suppressor Of Clear). These proteins define a family of docking proteins closely related to the insulin receptor substrate (IRS-1, IRS-2, IRS-3), fibroblast growth factor substrate (FRS2), linker of $\mathrm{T}$ cell (LAT), and downstream of kinase (Dok) families [1]. In contrast to adaptor proteins such as growth factor receptor bound protein 2 (Grb2) and Shc, which are usually smaller and often function as a molecular bridge between two proteins in the assembly of larger protein complexes, docking proteins contain a membrane-targeting region at the $\mathrm{N}$-terminus, binding sites for src homology 3 (SH3) domain-containing proteins, and multiple tyrosine phosphorylation sites that, when phosphorylated, function as binding sites for the src homology 2 ( $\mathrm{SH} 2$ ) domains of a variety of effectors. Consequently, the docking proteins are significantly larger than adaptor proteins. In addition, docking proteins usually contain one or more moieties that mediate their recruitment to plasma membranes through protein-protein or protein-lipid interactions. Their multiple functional domains and large molecular size reflect the docking proteins' function as a platform for the assembly of signaling subsystems. Since there have been several excellent general reviews on Gab proteins to date [14], here we will focus on the role of Gab docking proteins in cardiovascular and inflammatory disorders.

\section{Identification of Gab Family Docking Proteins}

Gabl, the first of the three mammalian gab genes cloned to date, was originally identified as a Grb2-binding protein from a human glial tumor expression library and found to undergo tyrosine phosphorylation in response to stimulation by epidermal growth factor (EGF) and insulin [5]. It was also isolated as a c-Met-receptor interacting protein in a yeast twohybrid screen and as the major tyrosine-phosphorylated 
protein in cells transformed by the Tpr-Met oncogene $[6,7]$. Gab2 was cloned as a binding protein and a substrate of the $\mathrm{SH} 2$ domain-containing protein tyrosine phosphatase (SHP2) [8-10]. The Gab3 cDNA was cloned with the aid of the genome sequencing project, using a search strategy based on sequence similarities to Gab1 [11]. Although a putative Gab4 gene has been found in the human genome database, its expression pattern, signaling mechanism, and functional roles have not been characterized to date.

DOS is the only Gab homolog in Drosophila. It was identified as a potential substrate for the product of Corkscrew (Csw) [12], the Drosophila SHP2 ortholog, and independently in a screen for mutants that suppress the rough-eye phenotype of a hyper-activated sevenless allele [13]. SOC-1, the $C$. elegans homolog, was found in a screen for suppressors of hyperactive Egl-15 (an FGF receptor ortholog) signaling [14].

\section{Molecular Structure, Recruitment, and Phosphorylation of Gab Docking Proteins}

3.1. Molecular Structure. All Gab docking proteins share a highly conserved N-terminal Pleckstrin homology $(\mathrm{PH})$ domain, proline-rich segments in the central region, and multiple tyrosine residues within the potential binding motifs favored by various $\mathrm{SH} 2$ domain-containing signaling proteins (Figure 1) [1-4]. Mutagenesis and in vitro binding assays have demonstrated that a number of signaling molecules interact with Gab docking proteins (Figure 1).

3.2. Recruitment. Gab docking proteins utilize several different mechanisms to regulate their subcellular localization. First, the $\mathrm{PH}$ domain enables Gab proteins to translocate to plasma membrane patches enriched in phosphatidylinositol 3,4,5-triphosphate (PIP3), a product of phosphatidylinositol3 kinase (PI3K) [15-18]. Besides the PH domain, Gab docking proteins use at least two additional mechanisms for their recruitment to activated plasma membrane-associated receptors. The first mechanism has been demonstrated only for the interaction between Gabl and c-Met (the receptor for hepatocyte growth factor; HGF) [7]. A region in Gab1 (amino acids 450-532), termed the c-Met binding domain (MBD), interacts directly with the tyrosyl-phosphorylated cMet in response to stimulation with $\operatorname{HGF}[7,19-21]$. Both the activated kinase domain of c-Met and the MBD in Gab1 are involved in this direct interaction $[19,20]$. The minimal amino acid sequence sufficient for the direct interaction between Gabl and c-Met, termed the c-Met binding sequence (MBS), consists of 16 amino acids (486-501) [19, 20]. Since no other Gab docking proteins contain the MBS [22, 23], Gab2 interacts with activated receptors via the adaptor protein Grb2, which is also utilized as a secondary mechanism by the c-Met receptor to associate indirectly with Gabl. The importance of this indirect recruitment was revealed in knockout mice expressing a Gab1 mutant incapable of binding with Grb2: the phenotype was lethal [21].

3.3. Phosphorylation. Gab-mediated signal transduction is regulated by the site-specific tyrosine phosphorylation of the Gab proteins. Phosphorylated tyrosine residues provide docking sites for the SH2-domains of SHP2, the Crk adaptor protein, phospholipase $\mathrm{C}$ (PLC) $\gamma$, and the regulatory subunit of PI3K, p85 [2-4]. By recruiting various effectors with $\mathrm{SH} 2$ domains, Gab proteins not only promote signal transduction but also translate the receptor-evoked signals into distinct biological properties. Therefore, Gab family proteins function as a signaling platform for an entire signaling subsystem.

The best-characterized effector signaling pathway of Gab proteins is transmitted via the protein tyrosine phosphatase SHP2. SHP2 has two tandem N-terminal SH2 domains, which confer autoinhibition of the C-terminal phosphatase domain [24]. All mammalian Gab proteins, as well as the Drosophila DOS and C. elegans SOC-1, bind SHP2 (or its homologs), suggesting that the recruitment of SHP2 is an evolutionarily conserved feature of Gab family proteins [24]. Most Gab proteins contain two SHP2 binding sites, which act as a biphosphoryl tyrosine activation motif (BTAM) and bind both SH2 domains, which releases SHP2's autoinhibition [24, 25]. Therefore, SHP2 binding partners, including Gab proteins, may act not only as signaling platforms, but also as allosteric activators.

The functional significance of the Gab-SHP2 interaction has been extensively studied using mutants of Gab family proteins unable to bind SHP2 or its homologs. Mutant DOS bearing a $\mathrm{Y}$ to $\mathrm{F}$ mutation at either of the two CSW-binding sites is nonfunctional, and Sevenless signaling cannot rescue the lethal phenotype associated with DOS loss-of-function mutations [26, 27]. A Gabl mutant that is unable to bind SHP2 fails to transduce the signal for c-Met-dependent morphogenesis in MDCK cells and blocks the activation of extracellular signal-regulated kinase 1/2 (ERK1/2), MAP kinase upon stimulation with epidermal growth factor (EGF), HGF, or lysophosphatidic acid (LPA) [23, 25, 28, 29]. In endothelial cells, the recruitment of SHP2 to Gab1 not only regulates vascular endothelial growth factor- (VEGF-) induced migration, but also contributes to HGF-induced migration [30-32]. We also found that the Gab1-SHP2 interaction is involved in the activation of extracellular signal-regulated kinase 5 (ERK5) in gp130-dependent cardiomyocyte hypertrophy $[33,34]$. In addition, in certain cellular circumstances, the Gab-SHP2 complex positively regulates other downstream pathways, such as c-Kit-induced Rac activation and $\beta 1$-integrin-induced PI3K activation $[35,36]$.

These findings demonstrate that SHP2 is a crucial positive modulator for the activation of ERK1/2. Although the molecular mechanism underlying why the recruitment of SHP2 by Gab1 is required for the full activation of ERK1/2 is still not completely understood, two possible mechanisms have been proposed. First, SHP2 may dephosphorylate the recruitment site for the Src-inactivating kinase Csk on the transmembrane glycoprotein PAG/Cbp and paxillin, resulting in the enhanced activation of Src family kinases [37, 38]. Second, SHP2 may dephosphorylate the binding site for p120Ras-GAP on the activated receptors for EGF and on Gab1, thus inactivating the Ras-dependent signaling pathway $[37,39]$. 


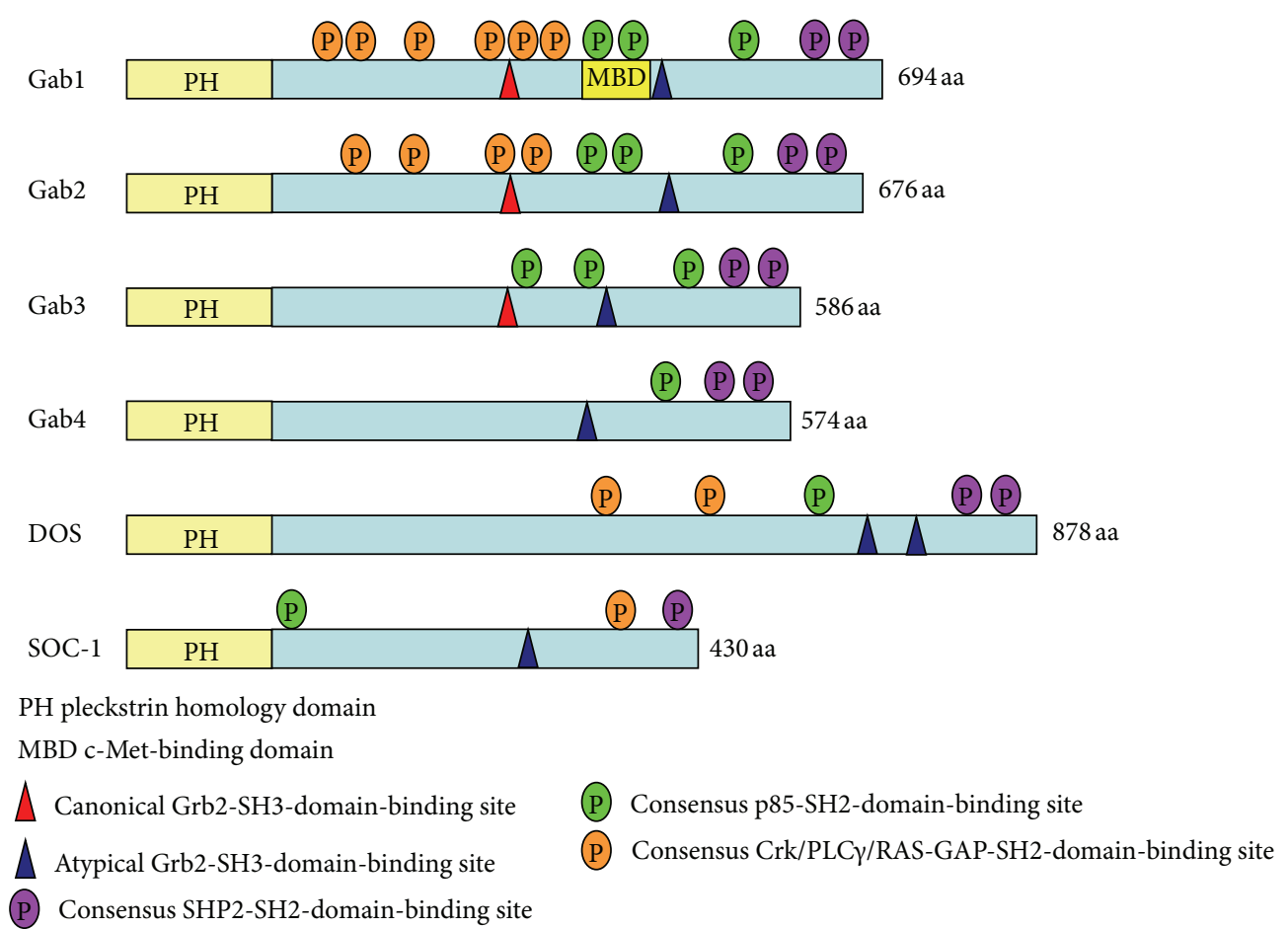

FIGURE 1: Schematic structures of Gab family docking proteins. Shown are the schematic domain structures of three human Gab proteins (Gab1-3), the putative human Gab4 protein, Drosophila DOS, and C. elegans SOC-1. All Gab proteins consist of a highly conserved N-terminal $\mathrm{PH}$ domain that is involved in membrane targeting. The central proline-rich regions mediate the association with $\mathrm{SH} 3$ domain-containing adaptor proteins such as Grb2. Consensus binding motifs favored by various SH2 domain-containing proteins such as SHP2, p85, Crk, and PLC $\gamma$ are indicated.

\section{Physiological Functions of Gab Proteins Revealed by Global Knockout or Knock-In Mice}

The presence of multiple gab genes in mammals suggests that the function of each Gab protein may be specialized or restricted to certain pathways or tissues. On the other hand, these gene products may be functionally redundant. Extensive analyses of the expression level of the gab genes by northern blot and RT-PCR have shed some light on this issue $[5,7,8,11]$. Gabl shows the broadest expression and greatest abundance: it is found in almost all tissues examined, including the brain, heart, liver, lung, kidney, pancreas, spleen, thymus, and uterus of the adult mouse, and is expressed at early developmental stages, such as in ES cells [11]. Although Gab2's expression is relatively weak in most tissue samples, compared with Gab1, it is abundantly expressed in hematopoietic progenitor cell lines, such as BAF3 and FDC-P1 $[5,7,8,11]$. The expression of Gab3 is also confined to the hematopoietic system $[5,7,8,11]$. Thus, the three mammalian gab genes have unique but overlapping expression patterns.

Consistent with Gabl's early and broad expression during development, Gabl-knockout $\left(\mathrm{Gabl}^{-/-}\right)$mice die in utero between embryonic days (E) 13.5 and 18.5 with developmental defects in the heart, placenta, skin, and skeletal muscle [40, 41]. In line with the close relationship between Gabl and
c-Met, $\mathrm{Gabl}^{-/-}$mice phenocopy most of the phenotypes of HGF- and c-Met-knockout mice, such as early embryonic lethality with placental defects, reduced liver size, and defects in the migration of muscle precursor cells [40, 41].

Gab1 knock-in mice carrying mutations in the SHP2 binding site show defects in muscle and placental development presumably directed by HGF/c-Met signaling, demonstrating a specific role for the Gab1-SHP2 complex in the migration of muscle progenitor cells [21]. Consistent with these findings, we found that the myogenic differentiation of $\mathrm{C} 2 \mathrm{C} 12$ cells induced by IGF-1 or low-serum conditions was strongly enhanced by the adenovirus-mediated overexpression of a mutated Gab1 $\left(\mathrm{Gab}^{\triangle \mathrm{SHP} 2}\right)$ incapable of binding SHP2, but inhibited by the overexpression of wildtype Gab1 [42]. This result suggests that Gabl negatively regulates myogenic differentiation through its association with SHP2. Taken together, these findings suggest that Gab1 plays a key role not only in the inhibition of myogenesis, but also in the maintenance of the undifferentiated state of mesenchymal cells, effected through the activation of SHP2. On the other hand, Gab1 knock-in mice carrying mutations in the p85 binding site show defects in EGF receptor-mediated embryonic eyelid closure and keratinocyte migration [21], and knock-in mice expressing a Gabl mutant lacking the Grb2 binding sites display an embryonic lethal phenotype and defects in liver, placenta, and craniofacial development [21]. These results support the idea that Gabl induces different 
biological responses through the recruitment of distinct effectors in vivo.

In contrast, Gab2-knockout $\left(\mathrm{Gab}^{-/-}\right)$mice are viable, generally healthy, and have an apparently normal life span. Although Gab2 was initially believed to be essential for the development of various hematopoietic lineages through its association with SHP2 [43], steady state hematopoiesis is largely normal in Gab2 $2^{-/-}$mice $[44,45]$. However, Gab2 $2^{-/-}$ mice exhibit a drastic phenotype in mast cell functioning [44]. Mast cells are major players in allergic responses, and $\mathrm{Gab}^{-/-}$mice have severe defects in their response to passive allergic challenge; their mast cells display defects in degranulation and cytokine gene expression in response to the activation of FceRI, the high-affinity IgE receptor. The defective activation of Gab2 $2^{-/-}$mast cells is ascribed mainly to their failure to induce PI3K activation. Furthermore, $\mathrm{Gab}^{-/-}$mice show decreased numbers of mast cells in various tissues, including the skin and stomach, because of weakened c-Kit signaling [45]. These findings suggest that Gab2, which is often upregulated in inflammatory disease, might be an important target for novel therapies against inflammation and allergy [46].

$\mathrm{Gab}^{-/-}$mice also exhibit an osteopetrotic phenotype that is attributed to the role of Gab2 in regulating RANK(receptor activator of nuclear factor- $\kappa-\mathrm{B}-$ ) dependent signaling [47]. Gab2 associates with RANK and mediates the RANK-induced activation of NF- $\kappa \mathrm{B}, \mathrm{AKT}$, and JNK. Bone homeostasis is determined by an intricate balance between the anabolic action of mesenchymal osteoblasts and the catabolic action of osteoclasts. Consistent with Gab2's pivotal role in the differentiation of a variety of hematopoietic lineages $[43,45], \mathrm{Gab}^{-/-}$mice exhibit defective osteoclast differentiation, resulting in decreased bone resorption and a subsequent systemic increase in bone mass [47]. In addition, Gab2 has a crucial role in the differentiation of human progenitor cells into osteoclasts [47].

To dissect the Gab2-dependent signaling pathways required for the degranulation of mast cells in vivo, Nishida et al. established knock-in mice that express Gab2 mutated at the binding sites for either the PI3K regulatory subunit p85 or SHP2 [48]. They found that both binding sites of Gab2 are required for degranulation and the anaphylaxis response, but not for cytokine production or contact hypersensitivity. Interestingly, the PI3K, but not the SHP2, binding site turned out to be important for granule translocation during degranulation. In particular, the Fyn/Gab2/PI3K-signaling pathway activates a small GTPase, ADP-ribosylation factor (ARF)1, which regulates granule translocation. These results indicated that Fyn/Gab2/PI3K/ARF1-signaling is specifically required for granule translocation and the anaphylaxis response in mast cells [48].

No specific role has been identified to date for Gab3. $\mathrm{Gab3}^{-/-}$mice are healthy and viable, and no obvious phenotype was detected in Gab3 ${ }^{-/-}$macrophages, despite the strong upregulation of this protein during macrophage differentiation [49].

\section{Physiological Functions of Gab Proteins Revealed by Conditional Knockout Mice}

5.1. The Roles of Gab Proteins in Cardiomyocytes. Because the $\mathrm{Gab1}^{-/-}$phenotype is embryonically lethal in mice, several groups, including ours, have created conditional Gablknockout mice, to determine its physiological functions in adulthood [50-53]. Gab1 is exclusively expressed in the heart from E10.5 to 13.5 [40], indicating that it might have a specific role in the heart. Therefore, we created cardiomyocytespecific Gab1-knockout (GablCKO) mice, but these mice are viable and display no obvious cardiac phenotypes [52].

Since Gab1 and Gab2 are expressed in cardiomyocytes, we hypothesized that Gab2 might complement the loss of Gab1. We therefore created cardiomyocyte-specific Gab1/Gab2 double-knockout (DKO) mice by crossing GablCKO mice with $\mathrm{Gab}^{-/-}$mice [52]. Although the DKO mice were viable, they showed a high postnatal mortality rate with marked ventricular dilatation and reduced contractility. In addition, the DKO mice showed remarkable pathological phenotypes including endocardial fibroelastosis and a large number of abnormally dilated coronary vessels in the ventricles. Neuregulin-1 (NRG-1) and ErbB receptors, including ErbB2 and ErbB4, comprise an important signaling pathway for heart development and the maintenance of heart function in adulthood. The NRG-1-induced activation of ERK1/2 and AKT were observed in the hearts of control, GablCKO, and $\mathrm{Gab}^{-/-}$mice, but not of DKO mice. These results suggest that Gab1 and Gab2 share a critically redundant role in NRG-1dependent signaling in cardiomyocytes (Figure 2).

To determine the effects of the DKO on gene expression, we performed a DNA microarray analysis of cardiac tissues, and found that NRG-1 upregulates the expression of the endothelium-stabilizing factor, angiopoietin-1 (Ang1), in the control mice, but not in the DKO mice [52]. Conventional Ang1-knockout mice show impaired development of myocardial trabeculae and vessel maturation [54], which are quite similar to the pathological abnormalities in the hearts of the DKO mice. Furthermore, the expression patterns of NRG-1 and ErbB are almost mirrored by those of Ang1 and Tie2, in the heart, suggesting that these two signaling pathways influence each other like a paracrine signaling circuit in the cardiac microenvironment [55]. These results suggest that the contributions of Gab1 and Gab2 to the crosstalk between NRG-1/ErbB and Ang1/Tie2 signaling are required for the maintenance of heart function (Figure 2).

5.2. The Role of Gab1 in Angiogenesis, Vascular Inflammation, and Atherosclerosis. Angiogenesis, the process of new blood vessel formation, is involved in many pathological settings, including ischemia, atherosclerosis, diabetes, and cancer [56]. It has been reported that Gab1 has a role in vascular endothelial growth factor- (VEGF-) dependent signaling in in vitro experiments using endothelial cells (ECs) [30, 31]. To reveal the in vivo role of Gab proteins in angiogenesis, we created endothelium-specific Gab1 knockout (GablECKO) mice [32]. The Gab1ECKO mice are viable and do not show any obvious defects in vascular development. We then subjected 


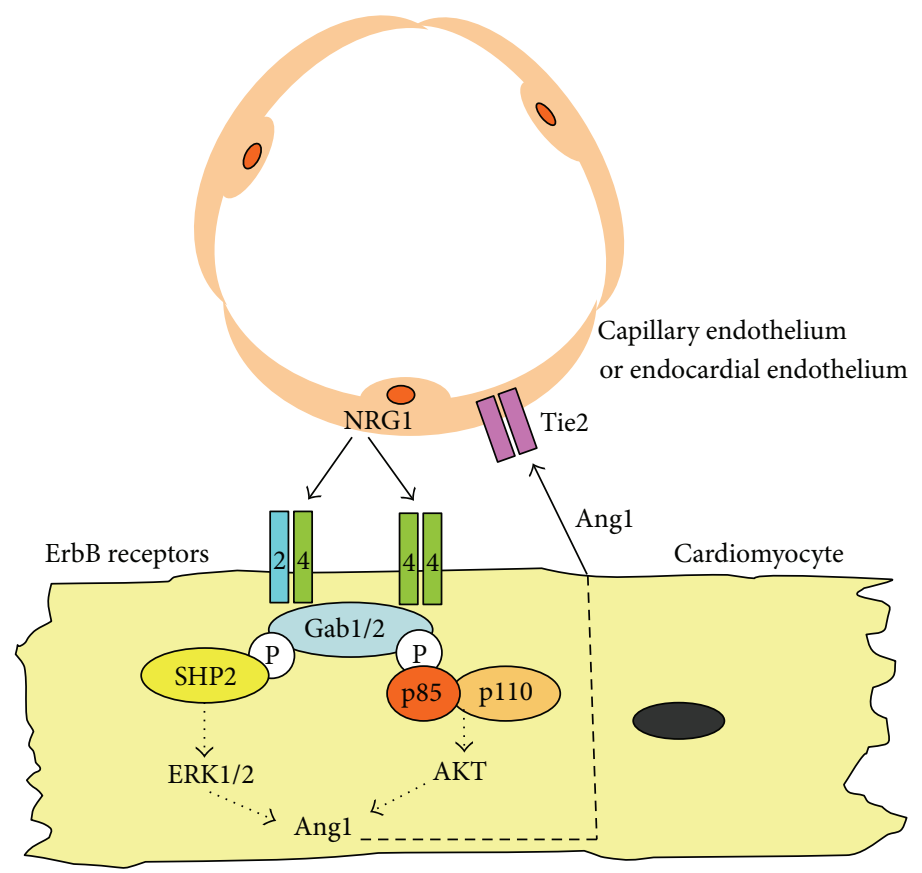

FIGURE 2: Schematic illustration of the roles of Gab docking proteins in the myocardium. Neuregulin-1 (NRG-1) shed from the capillary or endocardial endothelium in the heart activates the ErbB receptors expressed on cardiomyocytes, leading to the tyrosine phosphorylation of Gab1 and Gab2 and subsequent activation of ERK1/2 and AKT. NRG-1/ErbB-Gab1/Gab2 signaling in the myocardium is directly required for the postnatal maintenance of myocardial function. Furthermore, NRG-1/ErbB-Gab1/Gab2 signaling indirectly contributes to the postnatal stabilization of capillary or endocardial endothelium via the upregulation of angiopoietin-1 (Ang1). Ang1 derived from myocardium activates the Tie2 receptor, which is expressed on the cardiac endothelial cells.

Gab1ECKO and Gab2 $2^{-/-}$mice to hindlimb ischemia (HLI) induced by unilateral femoral artery ligation. Intriguingly, impaired blood flow recovery and necrosis in the operated limb was observed in all the GablECKO mice, but not in the control (wild-type) or Gab2KO mice. In human ECs, we compared the effects of several angiogenic growth factors and found that HGF induces the most prominent tyrosine phosphorylation of Gabl and the greatest subsequent complex formation of Gab1 with both SHP2 and p85 [32]. The Gabl-SHP2 complex was required for both the HGF-induced migration and proliferation of ECs via the ERK1/2 pathway and the HGF-induced stabilization of ECs via ERK5. The Gabl-p85 complex also regulated the migration of ECs after HGF stimulation, and it regulates the activation of AKT [32]. A microarray analysis of HGFs effects on gene expression in ECs demonstrated that it upregulates angiogenesis-related genes such as Kruppel-like factor 2 (KLF2) and early growth response 1 via the Gab1-SHP2 complex in human ECs (Figure 3) [32]. Furthermore, gene transfer of VEGF, but not HGF, improved the blood flow recovery and ameliorated the limb necrosis after HLI in the GablECKO mice [32]. These results suggest that Gab1 is essential for postnatal angiogenesis after ischemia via PI3K HGF/c-Met signaling (Figure 3).

At the same time as our study, two other groups reported results on postnatal angiogenesis in Gab1ECKO mice using the HLI model $[57,58]$. Whereas Zhao et al. reported that endothelial Gabl is essential for HGF-dependent postnatal angiogenesis, a finding almost identical to ours [58], Lu et al. reported that Gab1 regulates postnatal VEGF-dependent angiogenesis through the protein kinase A- (PKA-) endothelial NOS (eNOS) pathway [57]. Together, these findings provided by three independent groups show that Gabl is a crucial signal transducer that unites the HGF-dependent and VEGFdependent signaling and angiogenesis in endothelial cells (Figure 3) [32, 57, 58].

Since the above findings led us to hypothesize that Gab1 might have a role in endothelial homeostasis, we intercrossed the GablECKO mice with apolipoprotein E (ApoE) knockout (ApoEKO) mice. Six-month-old male ApoEKO/Gab1ECKO and littermate control (ApoEKO) mice were treated with angiotensin II (AngII) via an osmotic infusion minipump for 4 weeks. After the AngII treatment, the ApoEKO/GablECKO mice showed significantly exacerbated atherosclerosis and aneurysm formation compared with control mice [59]. The production of proinflammatory cytokines in the aorta was also significantly greater in the ApoEKO/Gab1ECKO than in the control mice. Furthermore, the expression levels of KLF2 and KLF4, key transcription factors for endothelial homeostasis, were significantly reduced in the aortic endothelium of the ApoEKO/GablECKO mice compared with the control mice $[59,60]$. Consistent with the reduced expression of KLF2 and KLF4, both vascular cell adhesion molecule1 (VCAM-1) expression and macrophage infiltration of the aortic walls were enhanced in ApoEKO/GablECKO mice compared with the control mice [59, 60]. Taken together, these findings show that endothelial Gabl protects 


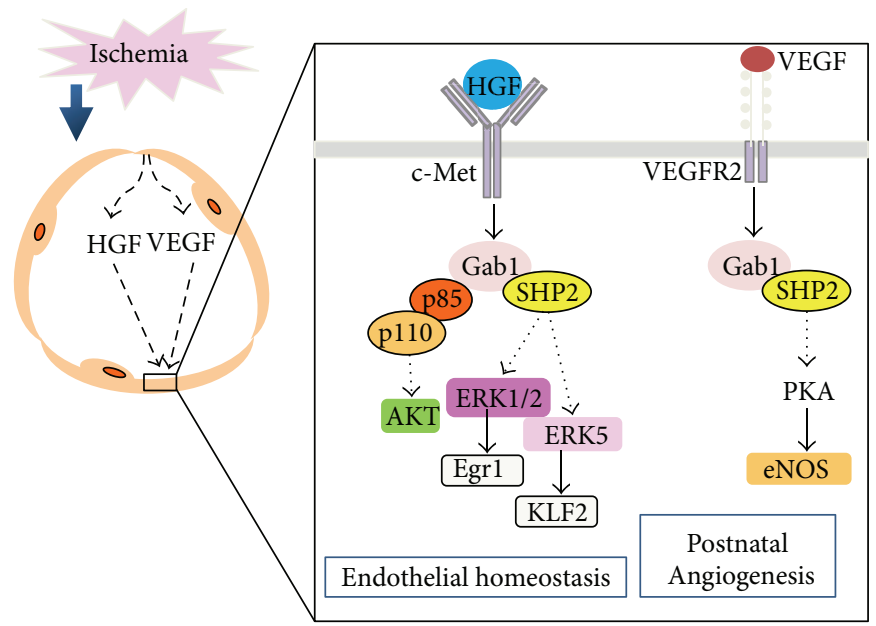

FiguRE 3: Schematic illustration of the role of Gabl in postnatal angiogenesis and endothelial homeostasis. Hypoxic tissues secrete growth factors, such as HGF and VEGF, which stimulate their specific receptors on endothelial cells (inset). The activation of c-Met receptors leads to the tyrosine phosphorylation of Gab1 and thereby to the subsequent complex formation of Gab1 with both SHP2 and p85. Whereas the formation of the Gab1-SHP2 complex is required for the activation of ERK1/2 and ERK5, the Gab1-p85 complex is essential for the activation of AKT in response to HGF. The ERK1/2 and ERK5 pathways contribute to the upregulation of early growth response 1 (Egrl) and Kruppel-like factor 2 (KLF2). On the other hand, activation of the VEGFR2 receptors leads to the tyrosine phosphorylation of Gab1, and to the subsequent formation of the Gab1-SHP2 complex, which causes the activation of protein kinase A (PKA) and endothelial nitric oxide synthase (eNOS). Collectively, current findings indicate that Gabl is an essential component of postnatal angiogenesis after ischemia.

the endothelium from AngII-dependent vascular inflammation and atherosclerosis in the ApoE-null background, presumably in association with the downregulation of KLF2 and KLF4 [59].

5.3. The Role of Gab1 in Liver Regeneration. Liver regeneration is a rapid and concerted response to injury, in which growth factor-evoked intracellular signals lead to the activation of various transcriptional factors, DNA synthesis, and hepatocyte proliferation. Liver-specific Gabl knockout (LGKO) mice exhibit defective liver regeneration after a two-thirds partial hepatectomy [50]. The defects in LGKO mice may be ascribed to the decreased proliferation of hepatocytes, due to the decreased activation of ERK1/2 and attenuated upregulation of immediate-early genes, such as c-fos, c-jun, and c-myc, after liver injury [50]. Interestingly, liver-specific SHP2-knockout mice phenocopy the defective liver regeneration of LGKO mice after partial hepatectomy, suggesting that Gab1 plays a critical role in liver regeneration via its association with SHP2 [50]. In addition, Gab1 negatively regulates the hepatic insulin-induced activation of AKT via the ERK1/2-mediated phosphorylation of IRS-1 on Ser612 [51]. Therefore, Gab1 is required not only for liver regeneration but also for the negative regulation of insulinmediated hepatic glucose homeostasis.

5.4. The Roles of Gab Proteins in Bone Homeostasis. The analysis of $\mathrm{Gab}^{-/-}$mice shows that Gab2 couples RANK to the downstream signaling essential for osteoclastogenesis, and that Gab2 has a negative regulatory role in osteoblast differentiation $[47,61]$. In contrast, osteoblast-specific Gabl-knockout mice display a low-bone-turnover osteopenic phenotype at 2 months of age, demonstrating an essential role for Gab1 in osteoblast functioning [53]. These results indicate that Gab1 and Gab2 have distinct functions in the maintenance of bone homeostasis: Gab1 in osteoblasts and Gab2 in osteoclasts.

\section{Gab Proteins in Human Cancers}

Gab proteins have been implicated in several hematological neoplasias and solid cancers, although only a few mutations have been reported in human Gab proteins to date. It is currently established that Gab proteins promote tumorigenesis by functioning as "accomplices" of certain oncoproteins or by amplifying signaling upon the Gab proteins' overexpression.

The chromosomal 11q13-14 locus containing the Gab2 gene is amplified in breast, ovarian, and gastric cancers and in acute myeloid leukemia (AML) [62-65]. Gab2 is overexpressed in estrogen receptor-positive cells [66], and a subset of breast cancers is driven by Gab2 overexpression coupled with RTK ErbB2 (also known as Neu or HER2) receptor signaling [62]. Consistent with these clinical results, Neel's group demonstrated that in the cultured human mammary epithelial cell line MCF-10A, the coexpression of wild-type Gab2, but not Gab2 ${ }^{\triangle \mathrm{SHP} 2}$ (incapable of binding SHP2) with ErbB2/Neu/HER2 resulted in an invasive growth phenotype [62]. They also revealed that NeuNT-transgeneevoked mammary tumorigenesis is potentiated in MMTVGab2 transgenic mice and attenuated in Gab2-deficient mice [62]. Similarly, Gab2's overexpression can potentiate metastatic melanomas [67]. Furthermore, myeloid progenitors from $\mathrm{Gab}^{-/-}$mice are resistant to transformation by $\mathrm{Bcr}-\mathrm{Abl}$, indicating that $\mathrm{Gab} 2$ is required to sustain the leukemogenesis evoked by this oncogenic fusion protein in 
a model of chronic myelogenous leukemia (CML) [68]. The phosphorylation of Y177 within the Bcr moiety results in the recruitment of the Grb2-Gab2 complex and the activation of downstream signaling via SHP2 and PI3K, which is essential for the cancer cells' enhanced proliferation and survival [68]. These results suggest that the Grb2-mediated recruitment of Gab2 to the oncogenic fusion protein $\mathrm{Bcr}-\mathrm{Abl}$ is a critical event for the induction of a CML-like disease. Gab2 is also important in the progression of other hematological neoplasias, such as juvenile myelomonocytic leukemia (JMML), acute myelocytic leukemia (AML), and acute lymphoblastic leukemia (ALL) $[65,69]$.

That Gabl plays a role in tumorigenesis is implied by its strong relationship with c-Met receptor signaling, since cMet is activated, mutated, or overexpressed in a wide range of cancers [19, 70,71]. Gab1 is also implicated as a mediator of EGFR-signaling-induced tumorigenesis in glioblastomas and intestinal adenomas $[72,73]$.

The elucidation of this direct linkage between Gab proteins and human cancers may contribute to the development of novel anticancer drugs in the future.

\section{Gab Proteins in Human Cardiovascular Diseases}

The neuro-cardiofacial-cutaneous (NCFC) syndromes consist of neurofibromatosis (NF), Noonan syndrome (NS), LEOPARD (multiple lentigines, electrocardiographic conduction abnormalities, ocular hypertelorism, pulmonary stenosis, abnormal genitalia, growth retardation, and sensorineuronal deafness) syndrome (LS), Costello syndrome, and cardiofacial-cutaneous syndrome. All of these syndromes are associated with autosomal-dominant germline mutations within either the core components (Ras, B-Raf, Raf-1, MEK) of the Ras-ERK1/2 pathway or its modulators (NF1, SHP2, SOS, and Spred). The resulting mutant proteins exhibit abnormal activities and disturbed overall fine-tuning of the Ras-ERK1/2 pathway (and to some extent of the Ras-PI3K pathway) $[74,75]$. Since the ERK1/2 pathway has a central role in both proliferation and differentiation, many processes in human development and organ maintenance are disturbed by its dysfunction, resulting in various clinical symptoms, such as a distinctive cranio-facial appearance, cardiac defects, musculocutaneous abnormalities, and mental retardation $[74,75]$. Germline missense mutations in the SHP2-encoding PTPN11 gene are seen in approximately $50 \%$ of NS cases; this observation contributed to the identification of PTPN11 as the most common target of somatic mutations in JMML [76, 77]. The most frequent JMML-associated mutation, E76 K, confers an enhanced catalytic activity on SHP2 and requires Gab2 for the transformation of primary murine myeloid progenitors [69]. This result demonstrates that Gab2 is an essential player in JMML and suggests that NS-associated SHP2 mutants may require Gab proteins similarly, as a recruitment tool.

Dominant-negative mutations of SHP2 are reported in LS patients, although NS patients usually carry constitutively active SHP2 mutations [78]. Intriguingly, the expression of
LS-associated SHP2 mutants with reduced catalytic activity in cultured cells significantly enhances the EGF-induced association of Gab1 with p85 [79]. This result suggests that LS-associated mutations in SHP2 might potentiate abnormal PI3K activation by blocking SHP2 from dephosphorylating the p85 recruitment sites on the Gab proteins. Collectively, these studies suggest that Gab proteins might exert an important role as "accomplices" of NCFC-associated SHP2 mutants in the pathogenesis of NCFC syndromes.

\section{Molecular Mimicry of Gab Proteins by a Bacterial Virulence Factor, CagA}

The CagA protein of the gastric pathogen Helicobacter pylori, a rod-shaped bacterium that infects the epithelial cells lining the stomach, has been described as functioning as a Gab-like protein [80]. The CagA protein is injected into the cytoplasm of gastric epithelial cells by the bacterium, whereupon it undergoes tyrosine phosphorylation by Src family kinases and $\mathrm{c}$-Abl on E-P-I-Y-A sequence motifs present in its C-terminal region $[81,82]$. Subsequently, CagA recruits $\mathrm{SH} 2$ domain-containing effector proteins such as SHP2 and Grb2, enabling CagA to effectively take over the signaling pathways that are normally regulated by Gab proteins. This process results in the rearrangement of actin cytoskeleton, cell scattering, and cell elongation, termed the "hummingbird" phenotype, which is reminiscent of the cellular response to Gab activation in cardiomyocytes and other cells [33, 83].

CagA has been categorized as a Gab mimic based on its ability to interact with partners of Gab and exert similar effects in human gastric cells [80]. Intriguingly, this concept of molecular mimicry is strongly supported by transgenic studies in Drosophila, demonstrating that a cagA transgene can rescue larval viability and photoreceptor development in mutant animals that lack DOS [84]. In addition, an epistasis analysis demonstrated that the complementation of DOS by CagA overexpression requires the expression of the SHP2 ortholog CSW [84]. Thus, these results revealed how CagA can mimic Gab/DOS proteins in vivo.

\section{Conclusion}

Since the discovery of Gab docking proteins, a little more than a decade ago, it has become evident that these proteins play critical roles in a variety of physiological processes as well as in disorders including cancer, inflammation, and cardiovascular diseases. Quite recently, a genome-wide association study conducted by Tamari's group identified Gabl as a candidate gene for adult asthma in the Japanese population [85]. Whereas the molecular mechanism underlying this association remains unclear, further studies focusing on Gab proteins will aid in elucidating the pathophysiology of this kind of bronchial asthma in the near future. Thus, the versatile functions of Gab docking proteins might extend beyond the original definition of a docking protein. Furthermore, through careful analyses of Gab docking proteins, as shown in this paper, we may be able to obtain a more detailed 
understanding of Gab-mediated cardiovascular diseases, cancers, and inflammation.

\section{Acknowledgments}

The authors thank their laboratory members for their valuable suggestions and discussion. This work was supported in part by a Grant-in-Aid from the Ministry of Education, Culture, Sports, Science, and Technology, Japan (to Y. Nakaoka and I. Komuro), and grants from the Takeda Medical Research Foundation; Daiichi-Sankyo Foundation of Life Science; Japan Foundation for Applied Enzymology; Kobayashi Magobe Memorial Medical Foundation; and the Mochida Memorial Foundation for Medical and Pharmaceutical Research (to Y. Nakaoka).

\section{References}

[1] F. U. Wohrle, R. J. Daly, and T. Brummer, "Function, regulation and pathological roles of the Gab/DOS docking proteins," Cell Communication and Signaling, vol. 7, article 22, 2009.

[2] H. Gu and B. G. Neel, "The "Gab" in signal transduction," Trends in Cell Biology, vol. 13, no. 3, pp. 122-130, 2003.

[3] Y. Liu and L. R. Rohrschneider, “The gift of Gab," FEBS Letters, vol. 515, no. 1-3, pp. 1-7, 2002.

[4] K. Nishida and T. Hirano, "The role of Gab family scaffolding adapter proteins in the signal transduction of cytokine and growth factor receptors," Cancer Science, vol. 94, no. 12, pp. 1029-1033, 2003.

[5] M. Holgado-Madruga, D. R. Emlet, D. K. Moscatello, A. K. Godwin, and A. J. Wong, "A Grb2-associated docking protein in EGF- and insulin-receptor signalling," Nature, vol. 379, no. 6565, pp. 560-564, 1996.

[6] E. D. Fixman, M. Holgado-Madruga, L. Nguyen et al., "Efficient cellular transformation by the Met oncoprotein requires a functional Grb2 binding site and correlates with phosphorylation of the Grb2-associated proteins, Cb1 and Gab1," Journal of Biological Chemistry, vol. 272, no. 32, pp. 20167-20172, 1997.

[7] K. M. Weidner, S. Di Cesare, M. Sachs, V. Brinkmann, J. Behrens, and W. Birchmeier, "Interaction between Gabl and the c-Met receptor tyrosine kinase is responsible for epithelial morphogenesis," Nature, vol. 384, no. 6605, pp. 173-176, 1996.

[8] H. Gu, J. C. Pratt, S. J. Burakoff, and B. G. Neel, "Cloning of p97/Gab2, the major SHP2-binding protein in hematopoietic cells, reveals a novel pathway for cytokine-induced gene activation," Molecular Cell, vol. 2, no. 6, pp. 729-740, 1998.

[9] K. Nishida, Y. Yoshida, M. Itoh et al., "Gab-family adapter proteins act downstream of cytokine and growth factor receptors and T- and B-cell antigen receptors," Blood, vol. 93, no. 6, pp. 1809-1816, 1999.

[10] C. Zhao, D. H. Yu, R. Shen, and G. S. Feng, "Gab2, a new pleckstrin homology domain-containing adapter protein, acts to uncouple signaling from ERK kinase to Elk-1," Journal of Biological Chemistry, vol. 274, no. 28, pp. 19649-19654, 1999.

[11] I. Wolf, B. J. Jenkins, Y. Liu et al., "Gab3, a new DOS/Gab family member, facilitates macrophage differentiation," Molecular and Cellular Biology, vol. 22, no. 1, pp. 231-244, 2002.

[12] R. Herbst, P. M. Carroll, J. D. Allard, J. Schilling, T. Raabe, and M. A. Simon, "Daughter of sevenless is a substrate of the phosphotyrosine phosphatase corkscrew and functions during sevenless signaling," Cell, vol. 85, no. 6, pp. 899-909, 1996.
[13] T. Raabe, J. Riesgo-Escovar, X. Liu et al., "DOS, a novel pleckstrin homology domain-containing protein required for signal transduction between sevenless and Ras1 in drosophila," Cell, vol. 85, no. 6, pp. 911-920, 1996.

[14] J. L. Schutzman, C. Z. Borland, J. C. Newman, M. K. Robinson, M. Kokel, and M. J. Stern, "The Caenorhabditis elegans EGL15 signaling pathway implicates a DOS-like multisubstrate adaptor protein in fibroblast growth factor signal transduction," Molecular and Cellular Biology, vol. 21, no. 23, pp. 8104-8116, 2001.

[15] S. J. Isakoff, T. Cardozo, J. Andreev et al., "Identification and analysis of $\mathrm{PH}$ domain-containing targets of phosphatidylinositol 3-kinase using a novel in vivo assay in yeast," EMBO Journal, vol. 17, no. 18, pp. 5374-5387, 1998.

[16] C. R. Maroun, D. K. Moscatello, M. A. Naujokas, M. HolgadoMadruga, A. J. Wong, and M. Park, "A conserved inositol phospholipid binding site within the pleckstrin homology domain of the Gabl docking protein is required for epithelial morphogenesis," Journal of Biological Chemistry, vol. 274, no. 44, pp. 31719-31726, 1999.

[17] C. R. Maroun, M. Holgado-Madruga, I. Royal et al., "The Gab1 $\mathrm{PH}$ domain is required for localization of Gabl at sites of cellcell contact and epithelial morphogenesis downstream from the Met receptor tyrosine kinase," Molecular and Cellular Biology, vol. 19, no. 3, pp. 1784-1799, 1999.

[18] G. A. Rodrigues, M. Falasca, Z. Zhang, S. H. Ong, and J. Schlessinger, "A novel positive feedback loop mediated by the docking protein Gab1 and phosphatidylinositol 3-kinase in epidermal growth factor receptor signaling," Molecular and Cellular Biology, vol. 20, no. 4, pp. 1448-1459, 2000.

[19] C. Birchmeier, W. Birchmeier, E. Gherardi, and G. F. Vande Woude, "Met, metastasis, motility and more," Nature Reviews Molecular Cell Biology, vol. 4, no. 12, pp. 915-925, 2003.

[20] L. S. Lock, M. M. Frigault, C. Saucier, and M. Park, "Grb2independent recruitment of Gab1 requires the C-terminal lobe and structural integrity of the met receptor kinase domain," Journal of Biological Chemistry, vol. 278, no. 32, pp. 3008330090, 2003.

[21] U. Schaeper, R. Vogel, J. Chmielowiec, J. Huelsken, M. Rosario, and W. Birchmeier, "Distinct requirements for Gabl in Met and EGF receptor signaling in vivo," Proceedings of the National Academy of Sciences of the United States of America, vol. 104, no. 39, pp. 15376-15381, 2007.

[22] L. S. Lock, C. R. Maroun, M. A. Naujokas, and M. Park, "Distinct recruitment and function of Gab1 and Gab2 in met receptormediated epithelial morphogenesis," Molecular Biology of the Cell, vol. 13, no. 6, pp. 2132-2146, 2002.

[23] U. Schaeper, N. H. Gehring, K. P. Fuchs, M. Sachs, B. Kempkes, and W. Birchmeier, "Coupling of Gab1 to c-Met, Grb2, and Shp2 mediates biological responses," Journal of Cell Biology, vol. 149, no. 7, pp. 1419-1432, 2000.

[24] B. G. Neel, H. Gu, and L. Pao, “The 'Shp'ing news: SH2 domaincontaining tyrosine phosphatases in cell signaling," Trends in Biochemical Sciences, vol. 28, no. 6, pp. 284-293, 2003.

[25] J. M. Cunnick, L. Mei, C. A. Doupnik, and J. Wu, "Phosphotyrosines 627 and 659 of Gabl constitute a bisphosphoryl tyrosine-based activation motif (BTAM) conferring binding and activation of SHP2," Journal of Biological Chemistry, vol. 276, no. 26, pp. 24380-24387, 2001.

[26] B. S. Bausenwein, M. Schmidt, B. Mielke, and T. Raabe, "In vivo functional analysis of the Daughter of Sevenless protein 
in receptor tyrosine kinase signaling," Mechanisms of Development, vol. 90, no. 2, pp. 205-215, 2000.

[27] R. Herbst, X. Zhang, J. Qin, and M. A. Simon, "Recruitment of the protein tyrosine phosphatase CSW by DOS is an essential step during signaling by the Sevenless receptor tyrosine kinase," EMBO Journal, vol. 18, no. 24, pp. 6950-6961, 1999.

[28] J. M. Cunnick, J. F. Dorsey, T. Munoz-Antonia, L. Mei, and J. $\mathrm{Wu}$, "Requirement of SHP2 binding to Grb2-associated binder1 for mitogen-activated protein kinase activation in response to lysophosphatidic acid and epidermal growth factor," Journal of Biological Chemistry, vol. 275, no. 18, pp. 13842-13848, 2000.

[29] C. R. Maroun, M. A. Naujokas, M. Holgado-Madruga, A. J. Wong, and M. Park, "The tyrosine phosphatase SHP-2 is required for sustained activation of extracellular signalregulated kinase and epithelial morphogenesis downstream from the met receptor tyrosine kinase," Molecular and Cellular Biology, vol. 20, no. 22, pp. 8513-8525, 2000.

[30] M. Dance, A. Montagner, A. Yart et al., "The adaptor protein Gab1 couples the stimulation of vascular endothelial growth factor receptor-2 to the activation of phosphoinositide 3kinase," Journal of Biological Chemistry, vol. 281, no. 32, pp. 23285-23295, 2006.

[31] M. Laramé, C. Chabot, M. Cloutier et al., "The scaffolding adapter Gabl mediates vascular endothelial growth factor signaling and is required for endothelial cell migration and capillary formation," Journal of Biological Chemistry, vol. 282, no. 11, pp. 7758-7769, 2007.

[32] W. Shioyama, Y. Nakaoka, K. Higuchi et al., "Docking protein gabl is an essential component of postnatal angiogenesis after ischemia via HGF/c-met signaling," Circulation Research, vol. 108, no. 6, pp. 664-675, 2011.

[33] Y. Nakaoka, K. Nishida, Y. Fujio et al., "Activation of gp130 transduces hypertrophic signal through interaction of scaffolding/docking protein Gab1 with tyrosine phosphatase SHP2 in cardiomyocytes," Circulation Research, vol. 93, no. 3, pp. 221229, 2003.

[34] Y. Nakaoka, W. Shioyama, S. Kunimoto et al., "SHP2 mediates gp130-dependent cardiomyocyte hypertrophy via negative regulation of skeletal alpha-actin gene," Journal of Molecular and Cellular Cardiology, vol. 49, no. 2, pp. 157-164, 2010.

[35] T. Brummer, D. Schramek, V. M. Hayes et al., "Increased proliferation and altered growth factor dependence of human mammary epithelial cells overexpressing the Gab2 docking protein," Journal of Biological Chemistry, vol. 281, no. 1, pp. 626637, 2006.

[36] M. Yu, J. Luo, W. Yang et al., "The scaffolding adapter Gab2, via Shp-2, regulates Kit-evoked mast cell proliferation by activating the Rac/JNK pathway," Journal of Biological Chemistry, vol. 281, no. 39, pp. 28615-28626, 2006.

[37] Y. Ren, S. Meng, L. Mei, Z. J. Zhao, R. Jove, and J. Wu, "Roles of Gab1 and SHP2 in paxillin tyrosine dephosphorylation and Src activation in response to epidermal growth factor," Journal of Biological Chemistry, vol. 279, no. 9, pp. 8497-8505, 2004.

[38] S. Q. Zhang, W. Yang, M. I. Kontaridis et al., " Shp2 regulates SRC family kinase activity and Ras/Erk activation by controlling Csk recruitment," Molecular Cell, vol. 13, no. 3, pp. 341-355, 2004.

[39] Y. M. Agazie and M. J. Hayman, "Molecular mechanism for a role of SHP2 in epidermal growth factor receptor signaling," Molecular and Cellular Biology, vol. 23, no. 21, pp. 7875-7886, 2003.
[40] M. Itoh, Y. Yoshida, K. Nishida, M. Narimatsu, M. Hibi, and T. Hirano, "Role of Gab l in heart, placenta, and skin development and growth factor- and cytokine-induced extracellular signalregulated kinase mitogen-activated protein kinase activation," Molecular and Cellular Biology, vol. 20, no. 10, pp. 3695-3704, 2000.

[41] M. Sachs, H. Brohmann, D. Zechner et al., "Essential role of Gab1 for signaling by the c-Met receptor in vivo," Journal of Cell Biology, vol. 150, no. 6, pp. 1375-1384, 2000.

[42] T. Koyama, Y. Nakaoka, Y. Fujio et al., "Interaction of scaffolding adaptor protein Gab1 with tyrosine phosphatase SHP2 negatively regulates IGF-I-dependent myogenic differentiation via the ERK1/2 signaling pathway," Journal of Biological Chemistry, vol. 283, no. 35, pp. 24234-24244, 2008.

[43] Y. Zhang, E. Diaz-Flores, G. Li et al., "Abnormal hematopoiesis in Gab2 mutant mice," Blood, vol. 110, no. 1, pp. 116-124, 2007.

[44] H. Gu, K. Saito, L. D. Klaman et al., "Essential role for Gab2 in the allergic response," Nature, vol. 412, no. 6843, pp. 186-190, 2001.

[45] K. Nishida, L. Wang, E. Morii et al., "Requirement of Gab2 for mast cell development and KitL/c-Kit signaling," Blood, vol. 99, no. 5, pp. 1866-1869, 2002.

[46] F. M. Batliwalla, E. C. Baechler, X. Xiao et al., "Peripheral blood gene expression profiling in rheumatoid arthritis," Genes and Immunity, vol. 6, no. 5, pp. 388-397, 2005.

[47] T. Wada, T. Nakashima, A. J. Oliveira-Dos-Santos et al., "The molecular scaffold Gab2 is a crucial component of RANK signaling and osteoclastogenesis," Nature Medicine, vol. 11, no. 4, pp. 394-399, 2005.

[48] K. Nishida, S. Yamasaki, A. Hasegawa, A. Iwamatsu, H. Koseki, and T. Hirano, "Gab2, via PI-3K, regulates ARF1 in FceRImediated granule translocation and mast cell degranulation," Journal of Immunology, vol. 187, no. 2, pp. 932-941, 2011.

[49] M. Seiffert, J. M. Custodio, I. Wolf et al., "Gab3-deficient mice exhibit normal development and hematopoiesis and are immunocompetent," Molecular and Cellular Biology, vol. 23, no. 7, pp. 2415-2424, 2003.

[50] E. A. Bard-Chapeau, J. Yuan, N. Droin et al., "Concerted functions of Gab1 and Shp2 in liver regeneration and hepatoprotection," Molecular and Cellular Biology, vol. 26, no. 12, pp. 4664-4674, 2006.

[51] E. A. Bard-Chapeau, A. L. Hevener, S. Long, E. E. Zhang, J. M. Olefsky, and G. S. Feng, "Deletion of Gab1 in the liver leads to enhanced glucose tolerance and improved hepatic insulin action," Nature Medicine, vol. 11, no. 5, pp. 567-571, 2005.

[52] Y. Nakaoka, K. Nishida, M. Narimatsu et al., "Gab family proteins are essential for postnatal maintenance of cardiac function via neuregulin-1/ErbB signaling," Journal of Clinical Investigation, vol. 117, no. 7, pp. 1771-1781, 2007.

[53] T. Weng, F. Mao, Y. Wang et al., "Osteoblastic molecular scaffold Gab1 is required for maintaining bone homeostasis," Journal of Cell Science, vol. 123, no. 5, pp. 682-689, 2010.

[54] C. Suri, P. F. Jones, S. Patan et al., "Requisite role of angiopoietin1, a ligand for the TIE2 receptor, during embryonic angiogenesis," Cell, vol. 87, no. 7, pp. 1171-1180, 1996.

[55] D. L. Brutsaert, "Cardiac endothelial-myocardial signaling: its role in cardiac growth, contractile performance, and rhythmicity," Physiological Reviews, vol. 83, no. 1, pp. 59-115, 2003.

[56] P. Carmeliet, "Angiogenesis in health and disease," Nature Medicine, vol. 9, no. 6, pp. 653-660, 2003. 
[57] Y. Lu, Y. Xiong, Y. Huo et al., "Grb-2-associated binder 1 (Gab1) regulates postnatal ischemic and VEGF-induced angiogenesis through the protein kinase A-endothelial NOS pathway," Proceedings of the National Academy of Sciences of the United States of America, vol. 108, no. 7, pp. 2957-2962, 2011.

[58] J. Zhao, W. Wang, C. H. Ha et al., "Endothelial Grb2-associated binder 1 is crucial for postnatal angiogenesis," Arteriosclerosis, Thrombosis, and Vascular Biology, vol. 31, pp. 1016-1023, 2011.

[59] K. Higuchi, Y. Nakaoka, W. Shioyama et al., "Endothelial Gab1 deletion accelerates angiotensin II-dependent vascular inflammation and atherosclerosis in apolipoprotein E knockout mice," Circulation Journal, vol. 76, no. 8, pp. 2031-2040, 2012.

[60] G. B. Atkins and M. K. Jain, "Role of Krüppel-like transcription factors in endothelial biology," Circulation Research, vol.100, no. 12, pp. 1686-1695, 2007.

[61] S. Itoh, F. Yoshitake, H. Narita, K. Ishihara, and S. Ebisu, "Gab2 plays distinct roles in bone homeostasis at different time points," Journal of Bone and Mineral Metabolism, vol. 25, no. 2, pp. 81-85, 2007.

[62] M. Bentires-Alj, S. G. Gil, R. Chan et al., "A role for the scaffolding adapter GAB2 in breast cancer," Nature Medicine, vol. 12, no. 1, pp. 114-121, 2006.

[63] L. A. Brown, S. E. Kalloger, M. A. Miller et al., "Amplification of 11 13 in ovarian carcinoma," Genes Chromosomes and Cancer, vol. 47, no. 6, pp. 481-489, 2008.

[64] S. H. Lee, E. G. Jeong, S. W. Nam, J. Y. Lee, N. J. Yoo, and S. H. Lee, "Increased expression of Gab2, a scaffolding adaptor of the tyrosine kinase signalling, in gastric carcinomas," Pathology, vol. 39, no. 3, pp. 326-329, 2007.

[65] A. Zatkova, C. Schoch, F. Speleman et al., "GAB2 is a novel target of 11q amplification in AML/MDS," Genes Chromosomes and Cancer, vol. 45, no. 9, pp. 798-807, 2006.

[66] R. J. Daly, H. Gu, J. Parmar et al., "The docking protein Gab2 is overexpressed and estrogen regulated in human breast cancer," Oncogene, vol. 21, no. 33, pp. 5175-5181, 2002.

[67] B. Horst, S. K. Gruvberger-Saal, B. D. Hopkins et al., "Gab2mediated signaling promotes melanoma metastasis," American Journal of Pathology, vol. 174, no. 4, pp. 1524-1533, 2009.

[68] M. Sattler, M. G. Mohi, Y. B. Pride et al., "Critical role for Gab2 in transformation by BCR/ABL," Cancer Cell, vol. 1, no. 5, pp. 479-492, 2002.

[69] M. G. Mohi, I. R. Williams, C. R. Dearolf et al., "Prognostic, therapeutic, and mechanistic implications of a mouse model of leukemia evoked by Shp2 (PTPN11) mutations," Cancer Cell, vol. 7, no. 2, pp. 179-191, 2005.

[70] J. P. Eder, G. F. Vande Woude, S. A. Boerner, and P. M. Lorusso, "Novel therapeutic inhibitors of the c-Met signaling pathway in cancer," Clinical Cancer Research, vol. 15, no. 7, pp. 2207-2214, 2009.

[71] K. Mood, C. Saucier, Y. S. Bong, H. S. Lee, M. Park, and I. O. Daar, "Gabl is required for cell cycle transition, cell proliferation, and transformation induced by an oncogenic Met receptor," Molecular Biology of the Cell, vol. 17, no. 9, pp. 37173728, 2006.

[72] G. S. Kapoor, Y. Zhan, G. R. Johnson, and D. M. O’Rourke, "Distinct domains in the SHP-2 phosphatase differentially regulate epidermal growth factor receptor/NF-kappa B activation through Gabl in glioblastoma cells," Molecular and Cellular Biology, vol. 24, no. 2, pp. 823-836, 2004.

[73] A. E. Moran, D. H. Hunt, S. H. Javid, M. Redston, A. M. Carothers, and M. M. Bertagnolli, "Apc deficiency is associated with increased Egfr activity in the intestinal enterocytes and adenomas of C57BL/6J-Min/+ mice," Journal of Biological Chemistry, vol. 279, no. 41, pp. 43261-43272, 2004.

[74] Y. Aoki, T. Niihori, Y. Narumi, S. Kure, and Y. Matsubara, "The RAS/MAPK syndromes: novel roles of the RAS pathway in human genetic disorders," Human Mutation, vol. 29, no. 8, pp. 992-1006, 2008.

[75] E. Denayer and E. Legius, "What's new in the neuro-cardiofacial-cutaneous syndromes?" European Journal of Pediatrics, vol. 166, no. 11, pp. 1091-1098, 2007.

[76] G. Chan, D. Kalaitzidis, T. Usenko et al., "Leukemogenic Ptpn11 causes fatal myeloproliferative disorder via cell-autonomous effects on multiple stages of hematopoiesis," Blood, vol. 113, no. 18, pp. 4414-4424, 2009.

[77] C. M. Niemeyer and C. P. Kratz, "Paediatric myelodysplastic syndromes and juvenile myelomonocytic leukaemia: molecular classification and treatment options," British Journal of Haematology, vol. 140, no. 6, pp. 610-624, 2008.

[78] M. I. Kontaridis, K. D. Swanson, F. S. David, D. Barford, and B. G. Neel, "PTPN11 (Shp2) mutations in LEOPARD syndrome have dominant negative, not activating, effects," Journal of Biological Chemistry, vol. 281, no. 10, pp. 6785-6792, 2006.

[79] N. Hanna, A. Montagner, W. H. Lee et al., "Reduced phosphatase activity of SHP-2 in LEOPARD syndrome: consequences for PI3K binding on Gabl," FEBS Letters, vol. 580, no. 10, pp. 2477-2482, 2006.

[80] M. Hatakeyama, "Helicobacter pylori CagA—a potential bacterial oncoprotein that functionally mimics the mammalian Gab family of adaptor proteins," Microbes and Infection, vol. 5, no. 2, pp. 143-150, 2003.

[81] M. Poppe, S. M. Feller, G. Römer, and S. Wessler, "Phosphorylation of Helicobacter pylori CagA by c-Abl leads to cell motility," Oncogene, vol. 26, no. 24, pp. 3462-3472, 2007.

[82] M. Selbach, S. Moese, C. R. Hauck, T. F. Meyer, and S. Backert, "Src is the kinase of the Helicobacter pylori CagA protein in vitro and in vivo," Journal of Biological Chemistry, vol. 277, no. 9, pp. 6775-6778, 2002.

[83] N. Tegtmeyer, S. Wessler, and S. Backert, "Role of the cagpathogenicity island encoded type IV secretion system in Helicobacter pylori pathogenesis," FEBS Journal, vol. 278, no. 8, pp. 1190-1202, 2011.

[84] C. M. Botham, A. M. Wandler, and K. Guillemin, "A transgenic Drosophila model demonstrates that the Helicobacter pylori CagA protein functions as a eukaryotic gab adaptor," PLoS Pathogens, vol. 4, no. 5, Article ID e1000064, 2008.

[85] T. Hirota, A. Takahashi, M. Kubo et al., "Genome-wide association study identifies three new susceptibility loci for adult asthma in the Japanese population," Nature Genetics, vol. 43, no. 9, pp. 893-896, 2011. 


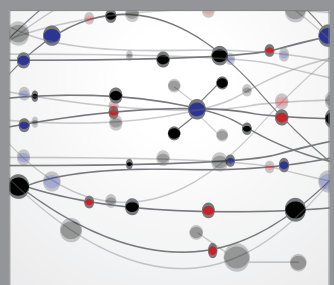

The Scientific World Journal
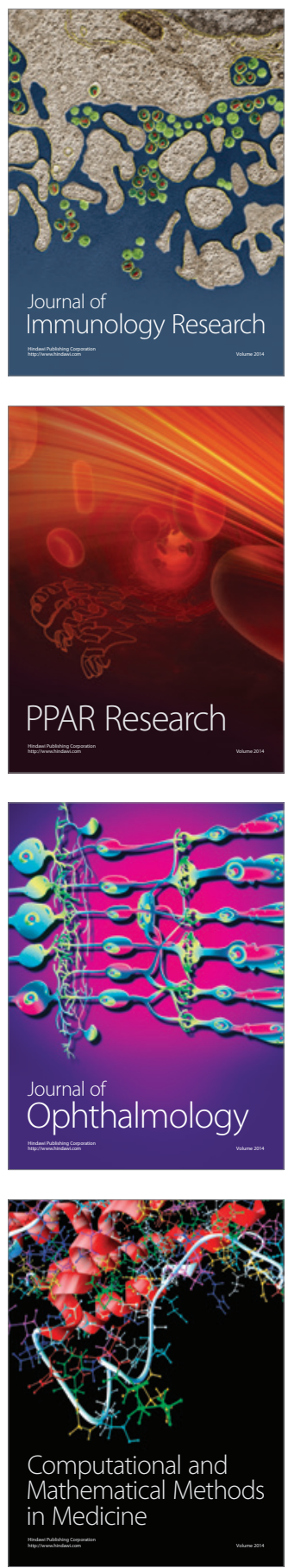

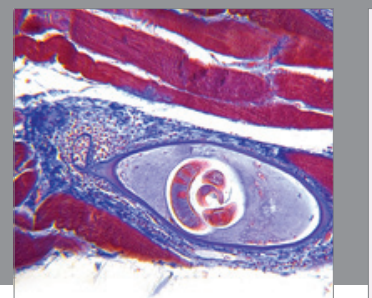

Gastroenterology

Research and Practice
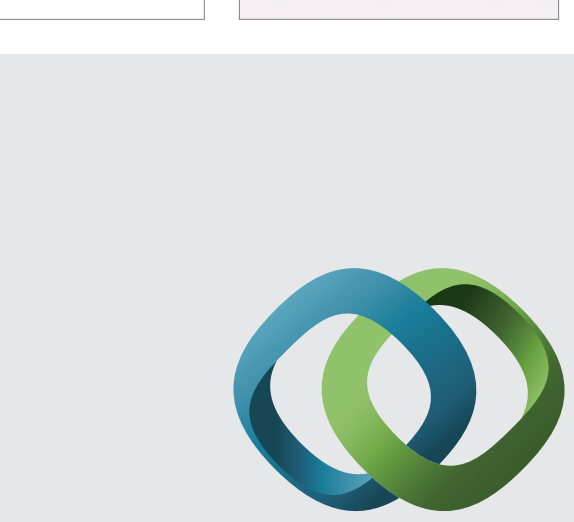

\section{Hindawi}

Submit your manuscripts at

http://www.hindawi.com
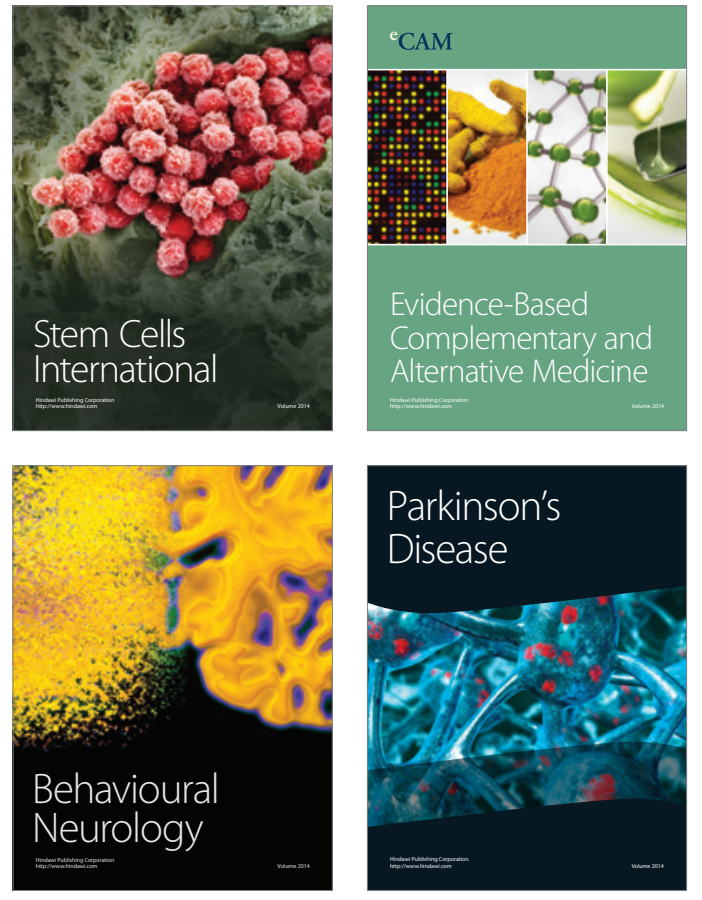
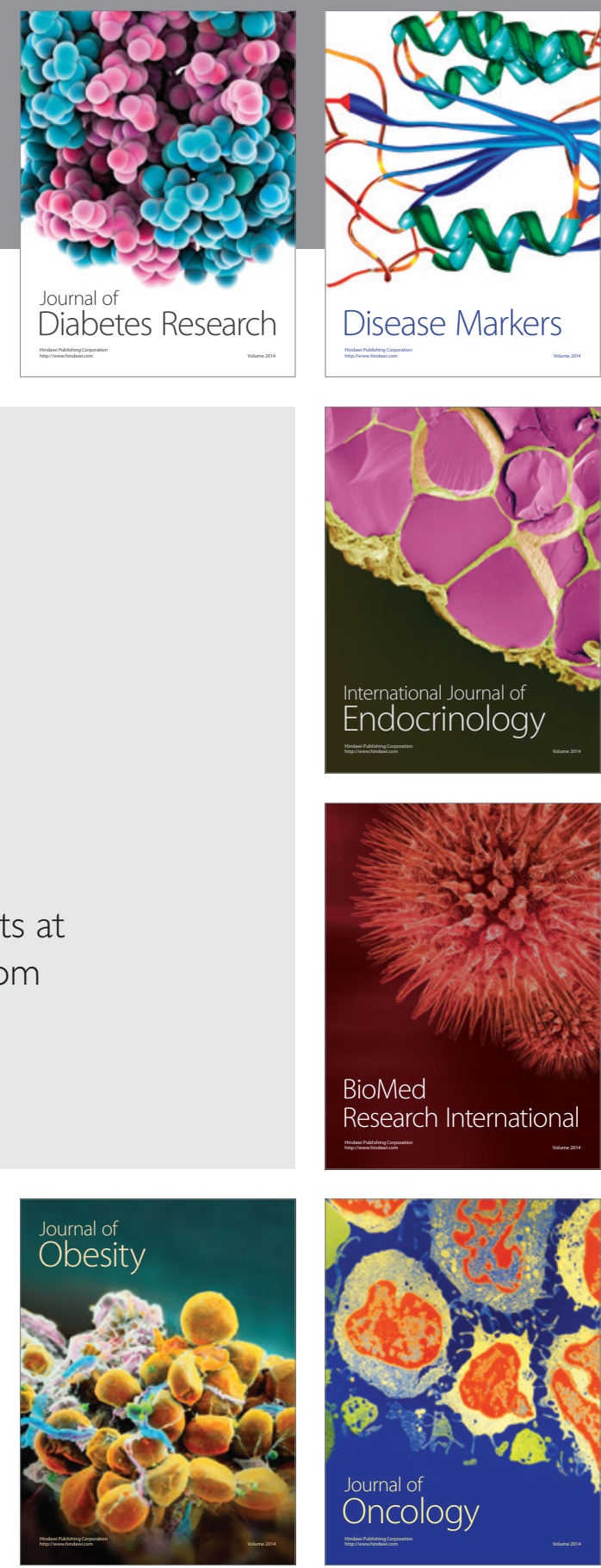

Disease Markers
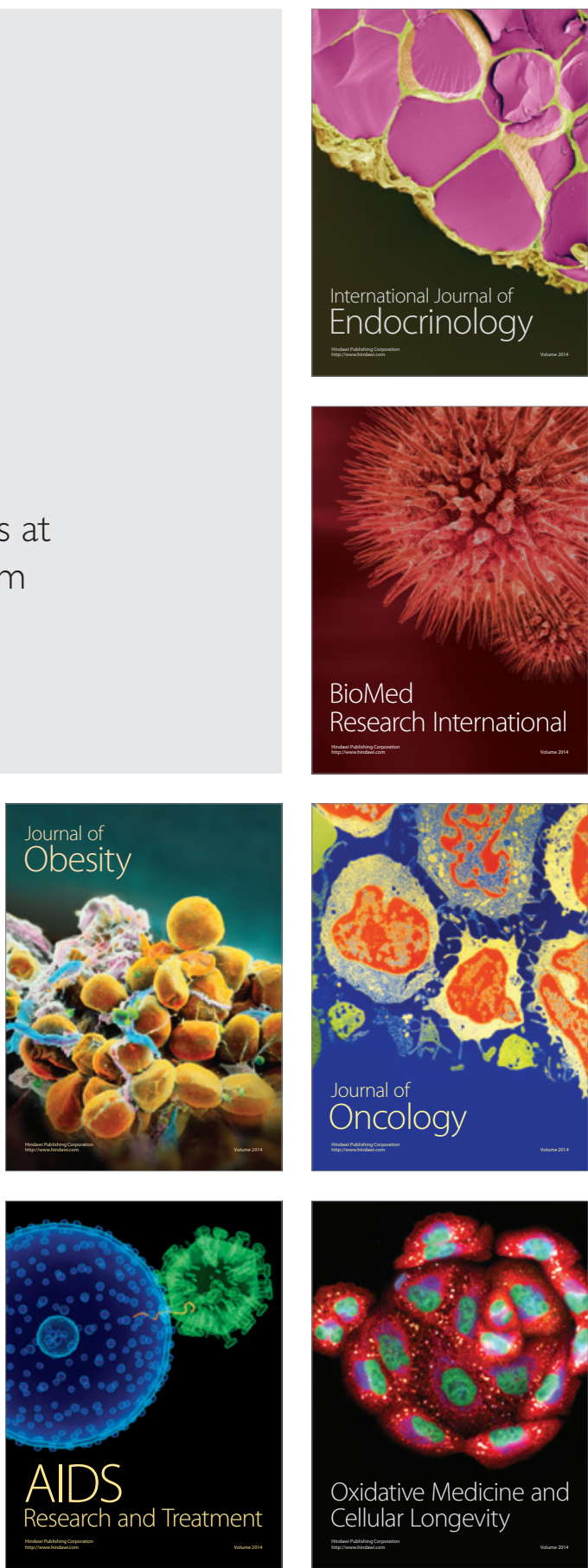